\title{
Collapsing Glomerulopathy in Transplanted Kidneys: Only a Tip of the Iceberg?
}

\section{Muhammed Mubarak}

Associate professor, Histopathology Department, Sindh Institute of Urology and Transplantation, Karachi-74200, Pakistan

Renal transplantation is the treatment of choice for patients with end-stage renal disease (ESRD) from a variety of causes. Although the short term results of renal transplantation have improved remarkably during the recent past, the long term outcomes have not improved to the same extent [1]. The long term success is marred by the occurrence of gradual onset of the often irreversible graft parenchymal scarring process, previously called as chronic allograft nephropathy (CAN), and now replaced by the term interstitial fibrosis/tubular atrophy (IF/ TA) by the Banff group [2]. The later complication is multifactorial in origin and is the final common pathway resulting from both immune and non-immune mechanisms of graft injury [1,2]. Among the non-immune causes, the occurrence of recurrent or de novo renal diseases, especially the glomerulopathies, is of particular concern, as the frequency of this complication tends to rise with increasing post transplantation duration and is one of the major causes of IF/TA in the long run [3].

Collapsing glomerulopathy (CG) is one of the distinctive patterns of proliferative renal parenchymal injury characterized histologically by the implosive, segmental and/or global wrinkling and collapse of the glomerular capillaries with sub-occlusion of the capillary lumena, marked hypertrophy and hypercellularity of podocytes, and severe tubulointerstitial damage [4]. It was initially described in native kidney biopsies [5-7], but more recently reports have appeared in the literature on its occurrence in the renal allograft biopsies [8]. Although initially thought to be caused by human immunodeficiency virus type 1 (HIV1), the lesion is now known to be triggered by a large variety of unrelated clinical conditions and etiologic agents in the native kidneys $[4,9]$. More importantly, the list of associated clinical conditions/ etiologic agents is growing day by day and encompasses the whole range of injurious agents from genetic, infections, drugs and chemicals, autoimmune, neoplastic, to ischemic diseases [4]. In addition, while the majority of the early reports on CG originated from centers in United States or Europe [5-7,10,11], more recently the lesion is also being increasingly recognized in the developing countries, implying a cosmopolitan distribution [12-15]. The data on CG in transplanted kidneys is still, however, sparse, and as alluded to earlier, most of the small case series that are available in literature have appeared from United States or Europe [16-21], but the lesion in this setting is also being increasingly reported from developing countries as well $[22,23]$. Although the etiological and clinical associations of CG in native kidneys are well characterized, the same in transplanted kidneys are still poorly described. The disease may recur post renal transplantation or may arise de novo. Majority of the reports on posttransplant CG have found de novo occurrence of this lesion [8,16,20]. Meehan et al. [8] were the first to report five cases of de novo CG after renal transplantation, which constituted $0.6 \%$ of all renal allograft biopsies and 3.2\% of their biopsies after 1993. They also found two cases of recurrent CG in their 892 allograft biopsies from 1079 patients [8]. Soon after, Stokes et al. [16] also from United States, reported six patients with de novo CG after renal transplantation and one patient with recurrent disease. The mean duration to diagnosis of CG post transplantation was 17.6 months in the series by Meehan et al. [8] and markedly longer (74 months) in the series by Stokes et al. [16], implying that CG, especially the de novo variety, may be a delayed complication of renal transplantation. More recently, Swaminathan et al. [20] studied in one of the largest series in renal transplant patients, 29 cases of posttransplant FSGS, 10 of collapsing focal segmental glomerulosclerosis (cFSGS) and 19 of non-collapsing FSGS (ncFSGS), and compared the presenting and follow-up data in the two groups. In the later series, there was no significant difference in age, gender, weight, delayed function, human leukocyte antigen (HLA) matching, acute rejection, and median time to diagnosis between cFSGS and ncFSGS groups. Deceased donor source was significantly more common in cFSGS group than in ncFSGS group. Patients with cFSGS presented with significantly more severe proteinuria and higher serum creatinine as compared with ncFSGS patients. The authors of the later study also did not find any evidence of viral etiology of the disease by in situ hybridization (ISH). From the developing world, Gupta et al. [22] from India have reported their experience on nine cases of CG in two years. The lesion constituted $3.5 \%$ of all their allograft biopsies, a rate slightly exceeding that of Meehan et al. [8] on biopsies after 1993. Although we have not systematically analyzed our data, but our findings are more or less concordant with those of the Indian study [23].

Although the clinical and laboratory parameters on presentation in some of the published series of posttransplant CG are similar to previously reported native kidney disease patients, there are a number of important differences. Only $10 \%$ to $14 \%$ of patients in these studies were black $[8,20]$, as compared with greater than $50 \%$ of patients in native renal biopsy series from the United States [4]. Patients also presented with markedly less proteinuria and a lower incidence of nephrotic syndrome (14\%) than patients with CG in the native renal biopsies [8]. The reasons for these differences in clinical presentation in these studies are not completely understood, but may partly be due to more aggressive biopsy practices in transplant patients with graft dysfunction or the attenuation of the disease process by immunosuppressive agents used in renal transplant recipients [20].

The pathogenesis of CG in renal transplant patients is as poorly understood as in native kidneys. An interesting hypothesis put forward by multiple investigators is that the pathogenesis of CG in renal transplant patients, especially of the de novo form, may be related to renal ischemia [8,10,16-21,23]. In Meehan et al. [8] series, four of the five patients had moderate to severe arteriolosclerosis and in Stokes et al. [16] series, five of the seven cases had moderate to severe vasculopathy that was related to cyclosporine (CsA) toxicity, thrombotic microangiopathy (TMA), and/or arteriolosclerosis. In Swaminathan et al. [20] series, the combined scores for arterial and arteriolar damage

Corresponding author: Muhammed Mubarak, Associate professor, Histopathology Department, Sindh Institute of Urology and Transplantation, Karachi-74200, Pakistan; Tel. 009221 9215752; Fax: 0092212726165 ; Email: drmubaraksiut@ yahoo.com

Received July 14, 2011; Accepted July 20, 2011; Published September 26, 2011

Citation: Mubarak M (2011) Collapsing Glomerulopathy in Transplanted Kidneys: Only a Tip of the Iceberg? J Transplant Technol Res 1:105e. doi:10.4172/2161$0991.1000105 e$

Copyright: (c) Mubarak M. This is an open-access article distributed under the terms of the Creative Commons Attribution License, which permits unrestricted use, distribution, and reproduction in any medium, provided the original author and source are credited. 
Citation: Mubarak M (2011) Collapsing Glomerulopathy in Transplanted Kidneys: Only a Tip of the Iceberg? J Transplant Technol Res 1:105e. doi:10.4172/2161-0991.1000105e

Page 2 of 2

were higher in cFSGS group as compared to ncFSGS. Similarly, five of the nine patients (55.5\%) in Indian series [22] showed moderate to marked vascular lesions in their biopsies. Recently, Nadasdy et al. [19] reported on three allograft nephrectomy specimens that showed a zonal distribution of CG that were topographically related to the obliterative vascular changes including chronic transplant arteriolopathy, acute vascular rejection, and TMA. More recently, three case reports have provided a more direct evidence for the causal relationship among CG and the subtotal/segmental infarction in three renal allograft patients [21]. The hypothesis that microvascular injury may be contributory to the pathogenesis of CG is also supported by studies in native kidneys [24]. A correlation between renal artery stenosis and FSGS also has been reported [25]. The above studies suggest that de novo CG in kidney allografts may be pathogenetically related to renovascular disease. A possible role for calcineurin-inhibitor (CNI) nephrotoxicity in the pathogenesis of de novo posttransplant FSGS has also been raised by many investigators in large studies of glomerular lesions among renal transplant patients [20]. CsA-based immunosuppression was more frequently employed among graft recipients with cFSGS compared with those with ncFSGS (50 vs $10.5 \%, \mathrm{P}=0.03$ ) while sirolimus-based immunosuppression was more common among ncFSGS cases (10 vs $32 \%, \mathrm{P}=0.17$ ) in the study by Swaminathan et al. [20]. The later study also found arteriolar hyalinosis more commonly in cFSGS cases than in ncFSGS patients.

CG after renal transplantation, as in native kidneys, has a uniformly poor prognosis. In the studies by Meehan et al. [8], and Stokes et al. [16], $100 \%$ and $71 \%$ of patients reached ESRD during the period of follow-up, respectively. The time to ESRD after the biopsy diagnosis averaged 9.8 months (range; 2-24 months) in Meehan et al.'s [8] series, and 2.6 months (range; 0 to 4 months) in Stokes et al. [16] series. Similarly, all patients with cFSGS progressed to graft loss within three years of diagnosis as compared with $40 \%$ graft loss in ncFSGS in Swaminathan et al.'s series [20]. In the Indian cohort, the prognosis was more favorable, possibly due to a shorter follow up duration in that study [22,23], as in the study of Stokes et al. [16].

There is no evidence based therapy for CG in general, and in the renal allograft patients, in particular. Currently employed regimens utilize the same strategy as used for ncFSGS and comprises of high dose steroids and other immunosuprresants, along with plasmapharesis. The success of these regimens is however limited. Newer molecular and genetic insights into the pathogenesis of CG may change this ominous outlook for this therapeutically resistant form of glomerular disease in both native and transplanted kidneys.

In conclusion, although many advances have been made in understanding the etiology and pathogenesis of CG especially in native kidneys, the data are still sparse in renal transplant patients. The recent advancements in the pathogenetic understanding are likely to open up new avenues for research into the rational therapy of this disease in both native and transplant kidneys.

\section{References}

1. Joosten SA, Kooten C, Sijpkens YW, Fijter JW, Paul LC (2004) The pathobiology of chronic allograft nephropathy: Immune-mediated damage and accelerated aging. Kidney Int 65:1556-1559.

2. Solez K, Colvin RB, Racusen LC, Sis B, Halloran PF, et al. (2007) Banff '05 Meeting report: Differential diagnosis of chronic allograft injury and elimination of chronic allograft nephropathy ('CAN'). Am J Transplant 7: 518-526.

3. Hariharan S, Adams MB, Brennan DC, Davis CL, First MR, et al. (1999)
Recurrent and de novo glomerular disease after renal transplantation: A Report from renal allograft disease registry (RADR)1,2. Transplantation 68: 635-641.

4. Albaqumi M, Barisoni $L$ (2008) Current views on collapsing glomerulopathy. $J$ Am Soc Nephrol 19: 1276-1281.

5. Weiss MA, Daquioag E, Margolin EG, Pollak VE (1986) Nephrotic syndrome, progressive irreversible renal failure, and glomerular "collapse." A new clinicopathologic entity? Am J Kidney Dis 7: 20-28

6. Detwiler RK, Falk RJ, Hogan SL, Jennette JC (1994) Collapsing glomerulopathy: a clinically and pathologically distinct variant of focal segmenta glomerulosclerosis. Kidney Int 45:1416-1424.

7. Valeri A, Barisoni L, Appel GB, Seigle R, D’Agati V (1996) Idiopathic collapsing focal segmental glomerulosclerosis: a clinicopathologic study. Kidney Int 50:1734-1746.

8. Meehan SM, Pascual M, Williams WW, Tolkoff-Rubin N, Delmonico FL, et al. 1998) De novo collapsing glomerulopathy in renal allografts 1 . Transplantation 65:1192-1197.

9. D'Agati V, Appel GB (1998) Renal pathology of human immunodeficiency virus infection. Semin Nephrol 18:406-421.

10. Meyrier AY (1999) Collapsing glomerulopathy: Expanding interest in a shrinking tuft. Am J Kidney Dis 33:801-803

11. Deegans JK, Steenbergen EJ, Borm GF, Wetzels JF (2008) Pathological variants of focal segmental glomerulosclerosis in an adult Dutch populationepidemiology and outcome. Nephrol Dial Transplant 23:186-192.

12. Kazi J, Mubarak M (2007) Collapsing FSGS: a need of awareness. J Pak Med Assoc 57:583.

13. Mubarak M, Kazi J (2010) Collapsing FSGS: a clinicopathologic study of 10 cases from Pakistan. Clin Exp Nephrol 14:222-227.

14. Agrawal V, Vinod PB, Krishnani N, Sharma RK (2008) A case of collapsing glomerulopathy associated with febrile illness. Indian J Pathol Microbio 51:509-511.

15. Nada R, Kharbanda JK, Bhatti A, Minz RW, Sakhuja V, et al. (2009) Primary focal segmental glomerulosclerosis in adults: is the Indian cohort different? Nephrol Dial Transplant 24:3701-3707.

16. Stokes MB, Davis CL, Alpers CE (1999) Collapsing glomerulopathy in rena allografts: a morphological pattern with diverse clinicopathologic associations. Am J Kidney Dis 33:658-666.

17. Clarkson MR, O'Meara YM, Murphy B, Rennke HG, Brady HR (1998) Collapsing glomerulopathy-recurrence in a renal allograft. Nephrol Dial Transplant 13 503-506.

18. Toth CM, Pascual M, Williams WWJr, Delmonico FL, Cosimi AB, et al. (1998) Recurrent collapsing glomerulopathy. Transplantation 65:1009-1010.

19. Nadasdy T, Allen C, Zand MS (2002) Zonal distribution of glomerular collapse in renal allografts: possible role of vascular changes. Hum Pathol 33:437-441.

20. Swaminathan S, Lager DJ, Qian X, Stegall MD, Larson TS, et al. (2006 Collapsing and non-collapsing focal segmental glomerulosclerosis in kidney transplants. Nephrol Dial Transplant 21:2607-2614.

21. Canaud G, Bruneval P, Noel L-H, Correas J-M, Audard V, et al. (2010) Glomerular collapse associated with subtotal renal infarction in kidney transplant recipients with multiple renal arteries. Am J Kidney Dis 55:558-565.

22. Gupta R, Sharma A, Agarwal SK, Dinda AK (2011) Collapsing glomerulopathy in renal allograft biopsies: a study of nine cases. Indian J Nephrol 21:10-13.

23. Mubarak M. Collapsing glomerulopathy in renal allograft biopsies: a study of nine cases. Letter to Editor. Indian J Nephrol (in press).

24. Greenberg A, Bastacky SI, Iqbal A, Borochovitz D, Johnson JP (1997) Focal segmental glomerulosclerosis associated with nephrotic syndrome in cholesterol atheroembolism: clinicopathological correlations. Am J Kidney Dis 29:334-344.

25. Thadhani R, Pascual M, Tolkoff-Rubin N, Nickeleit V, Colvin R (1996) Preliminary description of focal segmental glomerulosclerosis in patients with renovascular disease. Lancet 347:231-233. 